\title{
Housing Environment Affects Baseline Anxiety in Zebrafish
}

\author{
Bhavana Movva, Jacob W. Guerrant, Maxwell J. Jentsch, Allison N. Ogren, Julie L. Mustard, \\ Norbert W. Seidler
}

Laboratory of Neurobiology and Zebrafish Behavior Research, Division of Basic Sciences,

Kansas City University of Medicine and Biosciences, Kansas City, Missouri, USA

Email: nseidler@kcumb.edu

How to cite this paper: Movva, B., Guerrant, J.W., Jentsch, M.J., Ogren, A.N., Mustard, J.L. and Seidler, N.W. (2017) Housing Environment Affects Baseline Anxiety in Zebrafish. Journal of Behavioral and Brain Science, 7, 520-531.

https://doi.org/10.4236/jbbs.2017.711037

Received: October 4, 2017

Accepted: November 11, 2017

Published: November 14, 2017

Copyright $\odot 2017$ by authors and Scientific Research Publishing Inc. This work is licensed under the Creative Commons Attribution International License (CC BY 4.0).

http://creativecommons.org/licenses/by/4.0/

\begin{abstract}
Neuroscience researchers that wish to address compelling questions in psychopharmacology would benefit from the use of zebrafish, which have a behavioral repertoire that is rich and complex and that reflects many fundamental processes in humans, such as those that evoke anxiety. Zebrafish behavior is easily quantified in diverse test environments, but it is important to consider the conditions used to house the fish, prior to the behavioral testing, when designing experiments. Studies show that fish housed at high densities exhibit elevated cortisol levels as compared with fish housed at lower densities. The literature is less clear about the behavioral consequences of housing and handling. We examined the effects of housing (group versus paired) on several behavioral parameters. We observed that group-housed fish exhibited an anxious phenotype as evidenced by tank diving and meandering. Other more general indicators of movement and spatial navigation were unaffected by housing. This study focused on two specific housing environments, and the results support the need for continued research in this area to further elucidate the contributions of housing and handling on the subtleties of zebrafish behavioral markers.
\end{abstract}

\section{Keywords}

Zebrafish Behavior, Anxiety, Behavioral Phenotype, Animal Model

\section{Introduction}

Zebrafish is an excellent model for general neurobiology and for behavioral screening tests in psychiatric drug discovery [1]. Equipped with a complex behavioral repertoire, this model organism is amenable to development of protocols 
that assess a variety of behavioral parameters [2]. Zebrafish behavior is easily observed, video-recorded and quantified in a range of test environments [3]. We think that the conditions used to house the fish, prior to the behavioral testing, may be important when designing experiments. Protocols using zebrafish often involve choosing, removing and sub-dividing fish from a cohort originally kept in a large group tank of twenty or more fish derived from a single clutch. The transfer of fish to smaller group sizes, or to smaller tank sizes, may impact how they perform on behavioral tests [4]; and therefore, may be an important consideration when developing an experimental design, particularly involving response to test compounds. Additionally, the definition of a smaller number of fish per tank can vary from single-housed, or pair-housed, to higher numbers of fish. Likewise, tank sizes can vary, and therefore, densities of fish. We think that further research is necessary in identifying all aspects of housing as they relate to fish behavior.

Crowded housing conditions, for example, increase cortisol levels in zebrafish [5]. Fish that are housed in very large tanks (76 L) at a very high density (40 fish per liter) exhibit elevated cortisol levels as compared to those housed at a considerably lower density (0.25 fish per liter). Another study [4] supports this observation, demonstrating that group-housed fish (10 fish in $5 \mathrm{~L}$ ) show higher cortisol levels than individual-housed fish (1 fish in 1 L). Fish density, and not tank size, appears to be the dominant feature in this stress response. Group-stimulated cortisol elevation is present at a density as low as 4 fish per liter [5], suggesting that there may be a threshold fish density level, above which stress becomes a factor. An anxious behavioral phenotype would be expected to accompany this group-induced cortisol increase.

Tank-diving is a commonly used measure of anxiety-related behavior [6]. A fish, newly introduced to a novel tank, exhibits more bottom-dwelling behavior indicating anxiety. Experimentally, the specific size of the bottom compartment can vary among studies. Collymore and colleagues [7] designated the bottom half of their test tanks in measuring tank-diving. They compared bottom-dwelling in group-housed versus single-housed, either in barren tanks or in tanks enriched with plants. The researchers observed no differences among the four housing conditions; however, one could reason that these results may have been due to the relatively large size of the designated bottom zone. Smaller zone proportions can be used to define the lower compartment. When using a novel tank test with the lower third representing bottom-dwelling, Parker and colleagues [4] showed that group-housed fish spend more time in the bottom zone compared with pair-housed or individual-housed fish. The observed anxious behavioral phenotype of these group-housed fish is consistent with elevated cortisol.

Despite the lack of differences in bottom-dwelling among the four housing conditions, Collymore and colleagues [7], nevertheless, concluded that housing fish individually in a barren environment enhances anxiety and that enrichment 
with greenery may ameliorate stress. These conclusions were based on comparing the number of transition events across tank compartments in both the novel tank test as well as the light-dark test. This disparity in the literature suggests a need for further investigation into the effects of housing conditions on zebrafish behavior.

It would appear that previously under-valued aspects of experimental design may influence, in subtle ways, the data obtained, particularly when behavioral parameters are being measured. While testing conditions often receive the highest attention, methods used for housing and handling may be currently insufficiently emphasized or inadequately reported. In our study, we tested housing conditions as a factor that may influence baseline anxiety, to offer more observations to the literature that may provide guidance in designing experimental protocols where anxiety-related behaviors are being investigated.

\section{Methods}

\subsection{Zebrafish}

Adult wildtype $\mathrm{AB}$ zebrafish (Danio rerio), handled and bred using IACUC/IBC-approved protocols (\#764408 and \#764754), were used in this study. The single cohort population chosen for the project was derived from a line obtained as a gift from the Aquatics Facility at Stowers Institute for Medical Research (Kansas City, MO) and was housed and maintained in our facility until adulthood. Upon reaching adulthood, which occurs at 3 months post-fertilization (mpf), a cohort of 28 fish was transferred to a single $3 \mathrm{~L}$ group tank, where they remained until the start of the study. At this time, they were 510 days post-fertilization (dpf), chosen to represent middle-age subjects. The approximate human age equivalent of this cohort was calculated to be 47 years old, based on a previously published formula [8]. The zebrafish were kept on a Mini Z-Hab system (http://pentairaes.com/) under standard conditions $(\mathrm{pH}=7.3 \pm$ 0.4 ; conductivity $=650 \mu \mathrm{S} \pm 100$; temperature $28^{\circ} \mathrm{C} \pm 1.5^{\circ} \mathrm{C} ; 14 \mathrm{hr}: 10 \mathrm{hr}$ light/dark cycles). The housing environments described below were kept on the same rack on the system. Behavioral testing was conducted in a separate facility, a behavioral room approximately $10 \mathrm{~m}$ from the housing facility.

\subsection{Housing Environments}

This study tested two sets of zebrafish derived from the same cohort (Figure 1), which originally consisted of 28 conspecifics housed in the same $3 \mathrm{~L}$ group tank for approximately 420 days prior to initiation of the study. The first test set (6 zebrafish, mixed-gender) was randomly chosen from the original group-housing (GH) cohort. The GH fish, which were $510 \mathrm{dpf}$ at the time of testing, were individually video-recorded for behavioral analysis. These fish were not returned to the original $3 \mathrm{~L} \mathrm{GH}$ tank after testing. The second test set (10 zebrafish, mixed-gender), which was taken randomly from the remaining GH fish, was re-located into a paired-housing $(\mathrm{PH})$ environment that included five $1.5 \mathrm{~L}$ tanks 


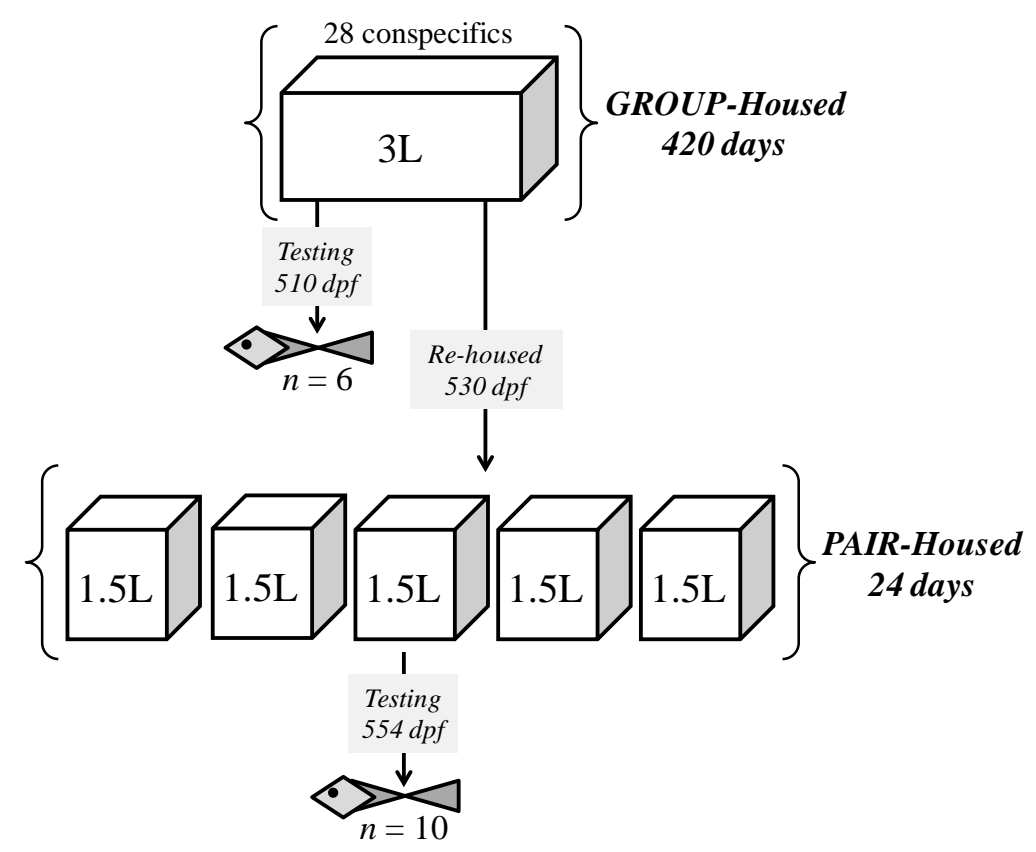

Figure 1. Experimental design. Schematic illustration of the two housing environments used in this study. All of the experimental subjects were derived from the same cohort of zebrafish. Each of the five pair-housed ( $\mathrm{PH})$ tanks was enriched with a plastic green plant.

each enriched with a plastic plant. The PH fish were allowed time to acclimate to their new surroundings for 24 days prior to behavioral testing, occurring at age $554 \mathrm{dpf}$. Following testing, the fish were euthanized.

\subsection{Neurobehavioral Testing}

Using the EthoVision XT 11.5 software (http://www.noldus.com/), the test fish were individually video-recorded in a neurobehavioral studio that consisted of a four-sided stadium blind made of white foam board (in cm: $40 \times 45 \times 70$; height, width, length). The $40-$ by- $70 \mathrm{~cm}$ foam boards represented the front and back walls of the stadium blind. A 1.5 L system tank was used as the "novel tank" and set on a small platform within the interior space. The test tank was positioned $9.5 \mathrm{~cm}$ from the back wall of the stadium blind, and the camera was directly outside the front wall at a distance of $30 \mathrm{~cm}$ from the tank, recording through a hole (diameter: $6.5 \mathrm{~cm}$ ) in the front wall. The stadium blind was flanked, at the top opening, with two light diffusers that held the light constant at 1200 lux \pm 20 . Laboratory overhead lights, in the behavioral room, were shut off, and there was no extraneous sound during the acclimation period and during video-recording.

Freshly prepared and pre-warmed $\left(26^{\circ} \mathrm{C}\right)$ environmental water $\left(\mathrm{Ev}-\mathrm{H}_{2} \mathrm{O}\right)$, containing $60 \mathrm{mg} / \mathrm{L}$ of standard marine salt and $87.5 \mu \mathrm{M}$ sodium bicarbonate $(p \mathrm{H} 7.3)$, was used in all steps involved in neurobehavioral testing. Each test fish was retrieved individually in a holding vessel $(900 \mathrm{~mL}$ spoutless beaker con-

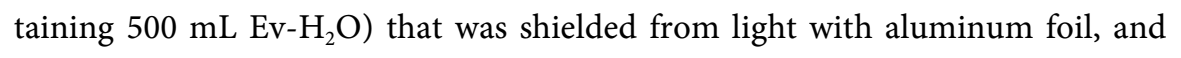
covered with parafilm to diffuse ambient light. The holding vessel was trans- 
ported to our behavioral room. The fish was kept in the holding vessel for $5 \mathrm{~min}$, undisturbed, prior to being transferred by decanting into the "novel tank", similar to that used in previous studies [4] [9], for immediate video-recording. The "novel tank" was prepped by adding $800 \mathrm{~mL}$ Ev- $\mathrm{H}_{2} \mathrm{O}$ prior to the fish transfer, making the final volume 1.3 $\mathrm{L}$ of Ev- $\mathrm{H}_{2} \mathrm{O}$. Each fish was video-recorded for 02:30 (min:s), allowing for two consecutive video-clips (each $1 \mathrm{~min}$ duration) to be analyzed per fish.

Following each video-recording, the test fish was removed, Ev- $\mathrm{H}_{2} \mathrm{O}$ disposed, and the test tank rinsed thoroughly with deionized water to remove any secreted substances (i.e. fear pheromones) that may influence the behavior of subsequent test fish. Camera resolution was $1280 \times 960$ with a frame rate of 25 per second. Detection settings were optimized using a non-test fish taken from the same cohort and was also not returned to the original housing tank. All video-recording occurred in the afternoon between 12:00 noon and 3:00 pm and was performed without a morning feeding.

\subsection{Analysis}

The video clips were analyzed by using EthoVision XT 11.5. Activity and movement in the overall arena, or swim area, was measured and compared, including total swim distance (in $\mathrm{cm}$ ), meandering (in deg per $\mathrm{cm}$ ), angular velocity (deg per s), and other mobility endpoints. The designated behavioral parameters in the two $60 \mathrm{~s}$ video clips were quantified for each fish: minute one from 00:15 to 01:15 (min:s); and minute two from 01:15 to 02:15, then averaged to obtain a single data point per fish. Most assessments were based on a center point that defined the movement and location of the fish's body. For meandering and angular velocity, head line direction established position. In some cases, the nose and tail points were used in addition to the center point in generating the results. Additionally, virtual zones were created and defined, as shown in Figure 2, that allowed us to measure time spent in specific zones as well as transitions across specific zones. The video image of the top edge of the "novel test tank" was calibrated to $28 \mathrm{~cm}$. While Figure 2 illustrates the tank in three-dimensional perspectives, the video-recordings were processed as containing two-dimensional zones. Zones 2, 3, 5, and 6 were equal in size: 9.5-by-5.5 cm (width and height). The upper foraging zone (Zone 1) was set to 6.0 -by- $4.25 \mathrm{~cm}$ (top and bottom width, respectively), and the lower foraging zone (Zone 4) to 4.25 -by-3.0 cm (top and bottom width, respectively). Zones 1 and 4 also had a height of $5.5 \mathrm{~cm}$. These zone designations were used to assess inter-zone movements thought to reflect foraging behaviors (unpublished observations). Figure 2(a) also allowed for measuring cumulative duration in the upper (Zones 1-3) versus lower zones (Zones 4-6) that can identify tank-diving behavior. Since the upper and lower zones were of equal height, we re-analyzed the video-recordings using a different configuration (Figure 2(b)) with the lower or deep zone having a height that is a third of the total water height in the tank. 


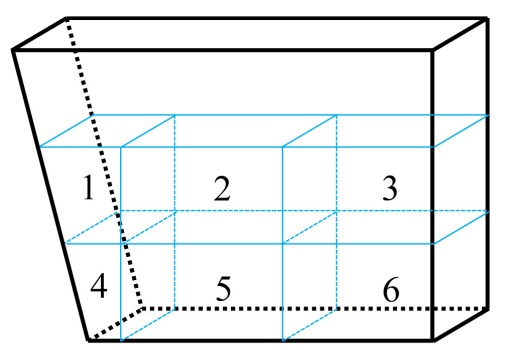

(a)

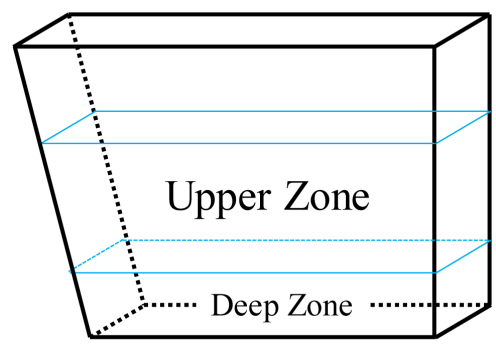

(b)

Figure 2. Illustration of "novel test tank" and the designated virtual zones. The overall swim area $(1300 \mathrm{~mL})$, or arena, was 25 -by- $22 \mathrm{~cm}$ (top and bottom width, respectively) and $11 \mathrm{~cm}$ (height). (a) Six virtual zones were designated with zones 1 and 4 considered the main foraging zones; (b) To test for tank diving, two virtual zones were designated with the bottom third representing the deep zone, $3.7 \mathrm{~cm}$ from the bottom edge.

\subsection{Statistical Tests}

Statistical tests were performed using Excel ${ }^{\circledR} 2010$ (https://www.microsoft.com/) or SigmaPlot 12.5 (https://systatsoftware.com/). Prior to all two sample comparisons, the Shapiro-Wilk test was applied to determine normal distributions. Tests for unequal variances were then automatically run. In some cases, F-tests were specifically done to compare sample variances. Non-parametric comparisons were done using Mann-Whitney Rank Sum Test, and Student's t-tests assessed parametric data. Statistical differences were defined as $p<0.05$ (i.e. $95 \%$ confident that there is a difference).

\section{Results}

We observed that there was no difference in total swim distance between GH and $\mathrm{PH}$ fish (Figure 3). The average swim distance for the GH fish was $285 \mathrm{~cm}$, compared to 354 for the $\mathrm{PH}$ fish. Both the normality test and equal variance test passed, but the calculated power of the performed test (0.195) was below the desired power of 0.800 , indicating that the negative results should be interpreted somewhat cautiously.

The range of total swim distance for the PH fish was approx. $250-500 \mathrm{~cm}$. 

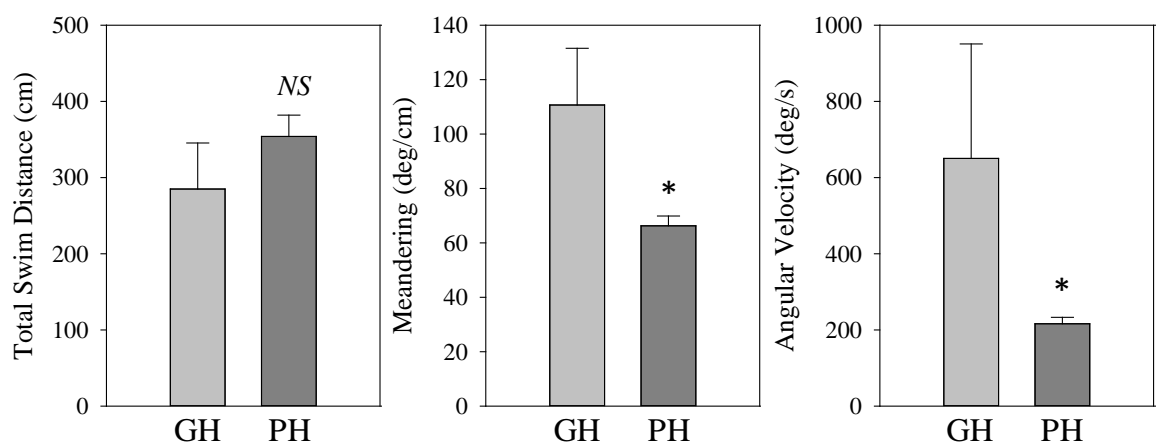

Figure 3. Comparisons of three key behavioral parameters. The total swim distance (LEFT panel) is a general indicator of movement. There were no statistical differences in this behavioral endpoint. Meandering (MIDDLE panel) and angular velocity (RIGHT panel), each representing an anxious phenotype, was significantly greater in the GH group compared to the $\mathrm{PH}$ group. Data are presented as sample means, and error bars represent SEM ( $N S$, not significant; asterisks, significant difference at $p<0.05)$.

This overlaps considerably with the range for GH fish, which was approx. 250 $420 \mathrm{~cm}$, with the exception of one subject in the GH sample group that swam a total distance of only $7.6 \mathrm{~cm}$, suggesting a type of rest behavior as a compensatory rebound to sleep deprivation. Perhaps this observation is a phenomenon associated with a socially-stratified subset of zebrafish in group-housing. While the numerical value appeared to be an obvious outlier, we kept all values from this test fish, as it may be an indicator of the variability of phenotype in the GH fish. Curiously, despite this apparent outlier, there was no difference in the variances of total swim distance between $\mathrm{GH}$ and $\mathrm{PH}$ fish. Therefore, with caution we conclude that the housing conditions in this study did not impact this particular behavioral indicator of overall movement (i.e. total swim distance).

Meandering, which is movement without a fixed path, was significantly greater in the GH group. Since the sample distribution did not conform to a normality test, a Mann-Whitney Rank Sum test was performed that indicated a statistical difference between the values of the two sample populations $(p=0.011)$. Angular velocity, a quantification of turn angle as a function of time, was also significantly greater in the $\mathrm{GH}$ group. The angular velocity data also did not show normal distribution of sample values, and thus, a non-parametric Rank Sum analysis was performed that also demonstrated a statistical difference ( $p=$ 0.045). For both parameters, an F-test showed that there was a significant difference in variances between $\mathrm{GH}$ and $\mathrm{PH}$ fish.

The EthoVision XT 11.5 program has the capability of differentiating and measuring the time spent in various states of mobility, as defined by the experimenter. The various levels of mobility were established by the amount of body fill change from one video still to the next. Mobility, here, is defined as the amount of body motion of a fish that is independent of the spatial displacement that occurs in swimming. With 25 frames per second, there are 1500 videos stills, from which to score frame-to-frame body fill changes in terms of detected pixels. Very high mobility was set as greater than $50 \%$ body fill change. High mobility 
was between $50 \%$ and $20 \%$ change, and average mobility was scored as less than $20 \%$. We observed that the GH fish exhibited a great time spent in the very high mobility state (Figure 4).

We measured the cumulative duration of time spent in an accelerated swim motion, as defined by reaching $20 \mathrm{~cm} / \mathrm{s}^{2}$ for at least 100 milliseconds. The frequency of reaching this acceleration threshold was also determined. This behavioral endpoint was unaffected by housing environment.

There was no difference in the cumulative duration of time spent in any particular zone (zones as defined in Figure 2(a)) between GH and PH fish. We looked at specific zone transitions that may be considered foraging-type behavior (i.e. swimming from Zone 5 to 4 to 1 ), but there were no differences observed. Even maximum zone alterations, measured as the total number of zones visited divided by the total number of zones available, were the same in the $\mathrm{GH}$ and PH fish. The six-zone arrangement allowed for measurement of tank diving, and thus, we calculated the amount of time spent in the upper half (Zones 1, 2 and 3; Figure 2(a)) versus the lower half (Zones 4, 5 and 6). There was no difference in cumulative duration of time spent in either of these compartments, as observed by comparing the total time spent in the lower half over the two minutes $(120 \mathrm{~s})$ : $\mathrm{GH}, 111.4 \mathrm{~s} \pm 14.98(\mathrm{M} \pm \mathrm{SD})$ versus $\mathrm{PH}, 83.5 \mathrm{~s} \pm 41.66(\mathrm{t}=1.559$, $\mathrm{df}=14$; two-tailed $p$-value $=0.141)$.

We decided to re-measure the time allocation in the tank by dividing the tank into a lower third and upper two-thirds (Figure 2(b)). When we assessed the video-recordings by scoring time spent in the deep tank zone, the data showed that the GH fish, relative to the PH fish, clearly favored this region and their behavior qualified as tank-diving (Figure 5).

In comparing cumulative duration in the deep zone, the tests for both normality and equal variance passed, and therefore, a t-test was run. While the

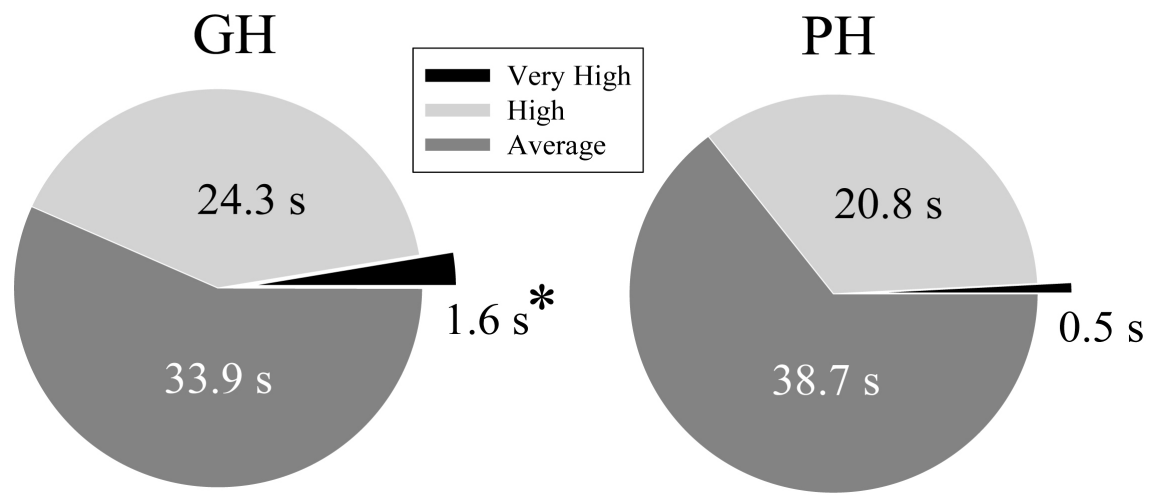

Figure 4. Housing environment affects the amount of time in very high mobility state. The pie graph illustrates the mean time spent (per minute) in three mobility categories: very high, high and average. The cut-off values: for very high mobility was greater than $50 \%$ body fill change between two succeeding video frames; for high, between $50 \%$ and $20 \%$; and for average mobility, less than $20 \%$ change in body fill. The very high mobility parameter was significantly different between the two groups; GH fish spent more time in that state than PH fish. Asterisk, $p<0.05$. 


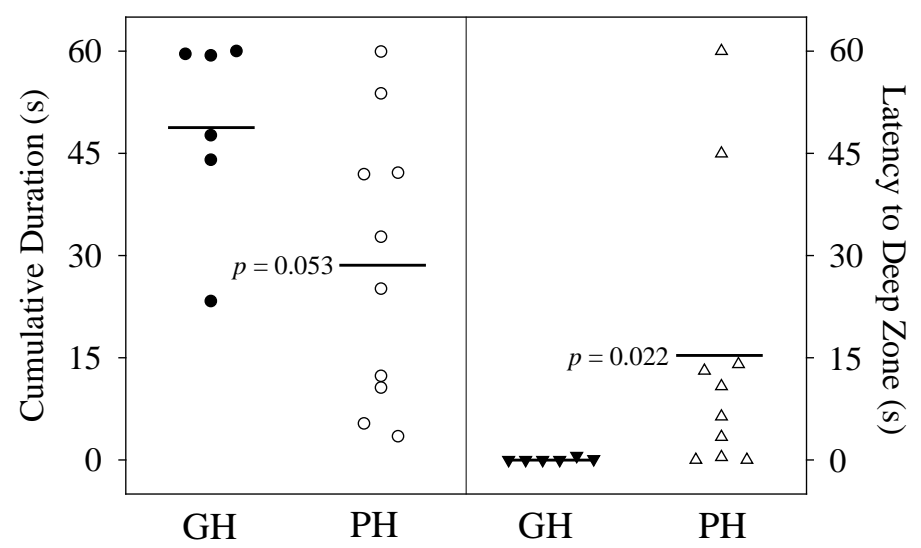

Figure 5. Group-housed fish exhibit tank-diving. Each data point represents the average per minute for each fish calculated over the first two minutes, given in seconds. Cumulative duration of time spent in the bottom third of the "novel test tank" is shown on the left, and latency (amount of time that elapsed before fish entered the bottom third of tank) is shown on the right. The data points from each subject are given (GH, filled symbols; $\mathrm{PH}$, open symbols) with the horizontal lines representing the means. The specific two-tailed $p$-values from t-tests are given.

two-tailed t-test yielded a $p$-value of 0.053 , a one-tailed approach may be justifiable considering that meandering was associated with the GH phenotype (Figure 3). The one-tailed $p$-value was 0.026 with a statistical power of $64 \%$, consistent with an anxious phenotype. In the analysis of latency to deep zone, since the normality test failed, a Mann-Whitney Rank Sum Test was applied. The comparative scores were particularly striking. All 6 subjects in the GH group were found in the deep zone at the start of each of the two one-minute measurements and $50 \%$ of the fish remained the entire time in the lower zone, suggesting that GH fish preferred this region of the tank, exhibiting more anxiety compared to $\mathrm{PH}$ fish.

\section{Discussion}

This study demonstrated that the simple act of re-housing fish from a tank with a high density (approx. 10 per liter) to one of low density (approx. 1 per liter) can significantly lower the behavioral markers of anxiety. This observation is consistent with previous findings that fish housed in large dense groups have higher cortisol levels than those individual-housed [4], or those fish housed in very low densities [5]. When studying target compounds that have psychiatric behavior impact, it would be important to consider the housing environment and therefore the baseline anxiety level of the test fish. In some circumstances, it may be pertinent to have a high baseline of anxiety, and in other situations, the researcher may want to have a very low level for baseline controls.

In our study, housing the fish in pairs diminished several behavioral markers of anxiety, including meandering and angular velocity. Both parameters were 
elevated in the GH fish. Meandering is a commonly used behavioral endpoint that can be evoked by anxiogenic challenges and responsive to anxiolytic agents [9]. Angular velocity, which is measured as the speed of the turn angle during swimming, is also used to assess an anxious phenotype and increases in response to acute ethanol withdrawal [10]. Both of these parameters, meandering and angular velocity, were scored in absolute terms, in that the total amount of turning was quantified over swim distance (i.e. degree per $\mathrm{cm}$ ) and over time (degree per s), respectively. The particular direction of turn, whether clockwise or counter-, was not indicated by \pm values and hence, not in relative terms that give a net direction of turning. Therefore, absolute meander and absolute angular velocity represent an aggregate sum of all turning movements of the fish's body, measured from the headline direction. We observed that the GH fish exhibited increased erratic turning movements compared to $\mathrm{PH}$ fish.

Modafinil, a wakefulness promoting drug, is shown to diminish thigmotaxis, a measure of anxiety, but does not affect meandering [11] under conditions described in that study, suggesting that the neural underpinnings that control the many behaviors associated with an anxious phenotype may be varied and complex. Anxiety is represented by behaviors in response to an individual's assessment of the potential risk presented by the environment [12]. Potential threats can be perceived or actual, and often associated with a new environment. Adverse stimuli may represent an anxiogenic challenge (i.e. pharmacological, psychological or "social", as implied in this study), evoking behaviors that may be associated with risk minimization. The risk assessment orientation of fish housed in groups may be influenced by the group dynamics and may require adjustment when individuals are isolated from their social complex.

The anxious phenotype of the GH fish appears to include movement-related behaviors (i.e. frequent bouts of turning, as indicated by the high levels of meandering and angular velocity, Figure 3; and jittery movement, as seen by greater thresholds of very high mobility, Figure 4) and tank-diving (Figure 5). These behaviors are consistent with the studies that show group housed fish having higher cortisol levels [4] [5]. Curiously, one of the GH fish exhibited a prolonged bout of sleep-like behavior. This anxiety-related behavior may indicate an under-recognized diversity of manifestations in the social dynamic. Many of the other behavioral endpoints that we examined (i.e. zone time, transitions and alterations) did not show a difference between the group- and pair-housed fish, suggesting that spatial navigation was unaffected. Also, the cumulative duration of time spent in an accelerated swim motion (i.e. $20 \mathrm{~cm} / \mathrm{s}^{2}$ for at least 100 milliseconds) was shown to be unaffected by housing environment. This parameter was previously examined, thought to be related to breathing [8], and in this study, provides a control for normal swim pattern.

There are several subtle features that are different in the housing environments that we compared in this study, and there are many similarities. GH and $\mathrm{PH}$ fish came from the same identical cohort derived from known breeding pairs 
and with the same fertilization date. This is important since it was observed that even slight genetic differences, such as among the different strains of zebrafish, can impact their baseline levels of anxiety [13]. Our design eliminated the likelihood of a genetic effect on the results that we obtained.

Another factor in our study, the actual date of testing (Figure 1), varied by about a month (44 days) between the GH and $\mathrm{PH}$ sample groups. Zebrafish age is an important consideration, as indicated by one study showed that age does impact anxiety parameters [14] but that study involved an age difference of six months (approx. 180 days). It is unlikely that 44 days would have a large effect and, furthermore, the effect would likely be more anxiety in the older fish, rather than the less anxiety that we observed in the slightly older $\mathrm{PH}$ fish.

The major differences between the $\mathrm{GH}$ and $\mathrm{PH}$ fish include the following: dimension/volume of tank; and the number of conspecifics that cohabitated the space. The fish density threshold, leading to increased anxiety, likely lies between the two groups examined. The behavioral testing was performed in a "novel test tank" that was a standard $1.5 \mathrm{~L}$ housing tank, similar to that used in other studies [4]. We think that the test tank offers a novelty to the $\mathrm{PH}$ fish, despite their being housed in similar $1.5 \mathrm{~L}$ tanks due to the stark changes in lighting conditions, stadium blind and absence of baffle, cover and green plant.

The added greenery to the $\mathrm{PH}$ tanks is typically done to minimize aggressive behavior observed in tanks with smaller numbers. In a study where single-housed fish kept in a barren environment were compared with those in an enriched (i.e. greenery added) environment, there was no difference observed in the novel tank diving or light-dark preference tests [7]. Given our experimental design we cannot completely rule out the contribution of the greenery, but in light of the existing data in the literature, we think that fish density plays the primary role in determining the difference in anxiety phenotype.

In summary, we conclude from our data that the primary independent variable, housing fish density ( $\mathrm{GH}$, or approx. 10 fish per liter versus $\mathrm{PH}$, approx. 1 fish per liter), affected the dependent variable of anxiety, as indicated by decreased behavioral markers in the $\mathrm{PH}$ sample, including meandering, angular velocity, bursts of high mobility, and tank-diving. In behaviors that are not related to anxiety (i.e. foraging and general swim behaviors), we observed similar levels of movement between the two groups, providing controls for non-specific changes in behavior.

\section{Acknowledgements}

The authors acknowledge the involvement of Daniel Quan, while a graduate student at KCU College of Biosciences, helping in the acquisition of the video-recordings. N.W.S. thanks Ashlee Dickerson and Gloria Ramirez for clerical assistance in preparation of the manuscript.

\section{References}

[1] Kokel, D. and Peterson, R.T. (2008) Chemo Behavioural Phenomics and Beha- 
viour-Based Psychiatric Drug Discovery in the Zebrafish. Briefings in Functional Genomics \& Proteomics, 7, 483-90. https://doi.org/10.1093/bfgp/eln040

[2] Kalueff, A.V., Gebhardt, M., Stewart, A.M., Cachat, J.M., Brimmer, M., Chawla, J.S., et al. (2013) Zebrafish Neuroscience Research Consortium. Towards a Comprehensive Catalog of Zebrafish Behavior 1.0 and Beyond. Zebrafish, 10, 70-86. https://doi.org/10.1089/zeb.2012.0861

[3] Kalueff, A.V., Stewart, A.M. and Gerlai, R. (2014) Zebrafish as an Emerging Model for Studying Complex Brain Disorders. Trends in Pharmacological Sciences, 35, 63-75. https://doi.org/10.1016/j.tips.2013.12.002

[4] Parker, M.O., Millington, M.E., Combe, F.J. and Brennan, C.H. (2012) Housing Conditions Differentially Affect Physiological and Behavioural Stress Responses of Zebrafish, as Well as the Response to Anxiolytics. PLoS One, 7, Article ID: e34992. https://doi.org/10.1371/journal.pone.0034992

[5] Ramsay, J., Feist, G., Varga, Z., Westerfield, M., Kent, M., et al. (2006) Whole-Body Cortisol Is an Indicator of Crowding Stress in Adult Zebrafish, Danio rerio. Aquaculture, 258, 565-574. https://doi.org/10.1016/j.aquaculture.2006.04.020

[6] Maximino, C., de Brito, T.M., da Silva, Batista, A.W., Herculano, A.M., Morato, S., et al. (2010) Measuring Anxiety in Zebrafish: A Critical Review. Behavioural Brain Research, 214, 157-171. https://doi.org/10.1016/j.bbr.2010.05.031

[7] Collymore, C., Tolwani, R.J. and Rasmussen, S. (2015) The Behavioral Effects of Single Housing and Environmental Enrichment on Adult Zebrafish (Danio rerio). American Association for Laboratory Animal Science, 54, 280-285.

[8] Mc Elroy, B., Mustard, J., Kamran, S., Jung, C., Bakken, K., Seidler, N.W. (2016) Modeling Post-Operative Cognitive Dysfunction in Zebrafish. Advances in Alzheimer's Disease, 5, 126-141. https://doi.org/10.4236/aad.2016.53009

[9] Cachat, J., Stewart, A., Utterback, E., Hart, P., Gaikwad, S., Wong, K., et al. (2011) Three-Dimensional Neurophenotyping of Adult Zebrafish Behavior. PLoS One, 6, Article ID: e17597. https://doi.org/10.1371/journal.pone.0017597

[10] Tran, S. and Gerlai, R. (2013) Time-Course of Behavioural Changes Induced by Ethanol in Zebrafish (Danio rerio). Behavioural Brain Research, 252, 204-213. https://doi.org/10.1016/j.bbr.2013.05.065

[11] Johnson, A. and Hamilton, T.J. (2017) Modafinil Decreases Anxiety-Like Behaviour in Zebrafish. Peer J, 5, Article ID: e2994. https://doi.org/10.7717/peerj.2994

[12] Graeff, F.G. and Zangrossi, J.H. (2010) The Hypothalamic-Pituitary-Adrenal Axis in Anxiety and Panic. Psychology \& Neuroscience, 3, 3-8. https://doi.org/10.3922/j.psns.2010.1.002

[13] Egan, R.J., Bergner, C.L., Hart, P.C., Cachat, J.M., Canavello, P.R., Elegante, M.F., et al. (2009) Understanding Behavioral and Physiological Phenotypes of Stress and Anxiety in Zebrafish. Behavioural Brain Research, 205, 38-44. https://doi.org/10.1016/j.bbr.2009.06.022

[14] Manuel, R., Gorissen, M., Stokkermans, M., Zethof, J., Ebbesson, L.O., van de Vis, H., et al. (2015) The Effects of Environmental Enrichment and Age-Related Differences on Inhibitory Avoidance in Zebrafish (Danio rerio Hamilton). Zebrafish, 12, 152-165. https://doi.org/10.1089/zeb.2014.1045 